\title{
Modelling the test, trace and quarantine strategy to control the COVID-19 epidemic in the state of São Paulo, Brazil
}

\author{
Marcos Amaku a, d, Dimas Tadeu Covas ${ }^{\mathrm{b}}$, Francisco Antonio Bezerra Coutinho a, \\ Raymundo Soares Azevedo Neto ${ }^{\mathrm{a}}$, Claudio Struchiner ${ }^{\mathrm{c}}$, Annelies Wilder- \\ Smith ${ }^{\mathrm{e}, \mathrm{f}, \mathrm{g}}$, Eduardo Massad ${ }^{\mathrm{a}, \mathrm{c},{ }^{*}}$ \\ a School of Medicine, University of Sao Paulo and LIM01-HCFMUSP, Sao Paulo, Brazil \\ b Instituto Butantan, Sao Paulo, Brazil \\ ' School of Applied Mathematics, Fundacao Getulio Vargas, Rio de Janeiro, Brazil \\ d School of Veterinary Medicine, University of Sao Paulo, Sao Paulo, Brazil \\ e Department Public Health and Clinical, Heidelberg Institute of Global Health, University of Heidelberg, Germany \\ ${ }^{\mathrm{f}}$ Medicine, Epidemiology and Global Health, Umeå University, SE-901 85, Umeå, Sweden \\ ${ }^{\mathrm{g}}$ Department of Disease Control, London School of Hygiene and Tropical, Medicine, UK
}

\section{A R T I C L E I N F O}

\section{Article history:}

Received 18 September 2020

Received in revised form 11 November 2020

Accepted 14 November 2020

Available online 20 November 2020

Handling editor: Dr. J Wu

\section{Keywords:}

COVID-19

SARS-CoV-2

Testing

Cost-analysis

Modelling

\begin{abstract}
A B S T R A C T
Testing for detecting the infection by SARS-CoV-2 is the bridge between the lockdown and the opening of society. In this paper we modelled and simulated a test-trace-andquarantine strategy to control the COVID-19 outbreak in the State of São Paulo, Brasil. The State of São Paulo failed to adopt an effective social distancing strategy, reaching at most 59\% in late March and started to relax the measures in late June, dropping to $41 \%$ in 08 August. Therefore, São Paulo relies heavily on a massive testing strategy in the attempt to control the epidemic.

Two alternative strategies combined with economic evaluations were simulated. One strategy included indiscriminately testing the entire population of the State, reaching more than 40 million people at a maximum cost of 2.25 billion USD, that would reduce the total number of cases by the end of 2020 by $90 \%$. The second strategy investigated testing only symptomatic cases and their immediate contacts - this strategy reached a maximum cost of 150 million USD but also reduced the number of cases by $90 \%$.

The conclusion is that if the State of São Paulo had decided to adopt the simulated strategy on April the 1st, it would have been possible to reduce the total number of cases by $90 \%$ at a cost of 2.25 billion US dollars for the indiscriminate strategy but at a much smaller cost of 125 million US dollars for the selective testing of symptomatic cases and their contacts.

(C) 2020 The Authors. Production and hosting by Elsevier B.V. on behalf of KeAi Communications Co., Ltd. This is an open access article under the CC BY-NC-ND license (http://creativecommons.org/licenses/by-nc-nd/4.0/).
\end{abstract}

\footnotetext{
* Corresponding author. School of Applied Mathematics, Fundacao Getulio Vargas, Rua Praia de Botafogo 190, Rio de Janeiro, CEP, 22250-900, RJ, Brazil. E-mail address: eduardo.massad@fgv.br (E. Massad).

Peer review under responsibility of KeAi Communications Co., Ltd.
} 


\section{Introduction}

Despite lockdowns in China, SARS-CoV-2 spread rapidly throughout the world by predicted routes of transportation (Bogoch et al., 2020) and spread faster than any other emerging infectious disease in recent decades (Angelo et al., 2019; Halstead \& Wilder-Smith, 2019; Tuite et al., 2019; Wilder-Smith, Chang, \& Leong, 2018). The first case of COVID-19 in Latin America was confirmed on February 26, 2020, in the São Paulo metropolis, the most populous city in the Southern hemisphere ( 12 million people) (Instituto Brasileiro de Geografia e Estatística, 2020). Travel reports and subsequent genetic analyses confirmed that the first detected infection was acquired via importation of the virus from Northern Italy (Candido et al., 2020; Jesus et al., 2020). Since then, Brazil has reported the largest number of cases in Latin America (4,238,446 reported cases and 129,522 deaths as of September 11, 2020) (Ministério da Saúde do Brasil, 2020). SARS-CoV-2 spread rapidly within Brazil (Carmo et al., 2020), often associated with urban centers with social deprivation (Souza et al., 2020) and has now been detected in the majority of the 27 federal states of Brazil. Using transmission pairs of SARS-CoV-2 reported to the Brazilian Ministry of Health, the mean and standard deviation for the serial interval was estimated to be 2.97 and 3.29 days respectively (Prete et al., 2020).

Social distancing, stay-at-home policies and discontinuation of mass gatherings up to complete lockdowns are important tenets of public health measures to mitigate the explosive growth of COVID-19 (Wilder-Smith \& Freedman, 2020). However, the most effective public health measures include liberal testing, prompt isolation of all test positive persons, contact tracing of all test positive cases and enforced quarantining of all contacts (Hellewell et al., 2020; Salathe et al., 2020).

Here we set out to model the number of infections and deaths depending on the timing and extent of testing and contact tracing in the State of Sao Paulo, the most populous state of Brazil with 44, 639, 899 inhabitants (Biblioteca Virtual do Estado de São Paulo, 2020).

The model

The model is a modified version of the classical SEIR type of models (Massad et al., 2020) and considers that the population is divided into several compartments, namely: susceptible individuals at time $t, S(t)$; tested susceptible individuals, $S_{T}(t)$; exposed individuals, $E(t)$; asymptomatic/oligosymptomatic individuals, $A(t)$; infectious individuals, $I(t)$; isolated infected individuals, $Q(t)$; hospitalized individuals, $H(t)$; individuals with severe disease hospitalized in intensive care units (ICU), $G(t)$; and recovered individuals, $R(t)$.

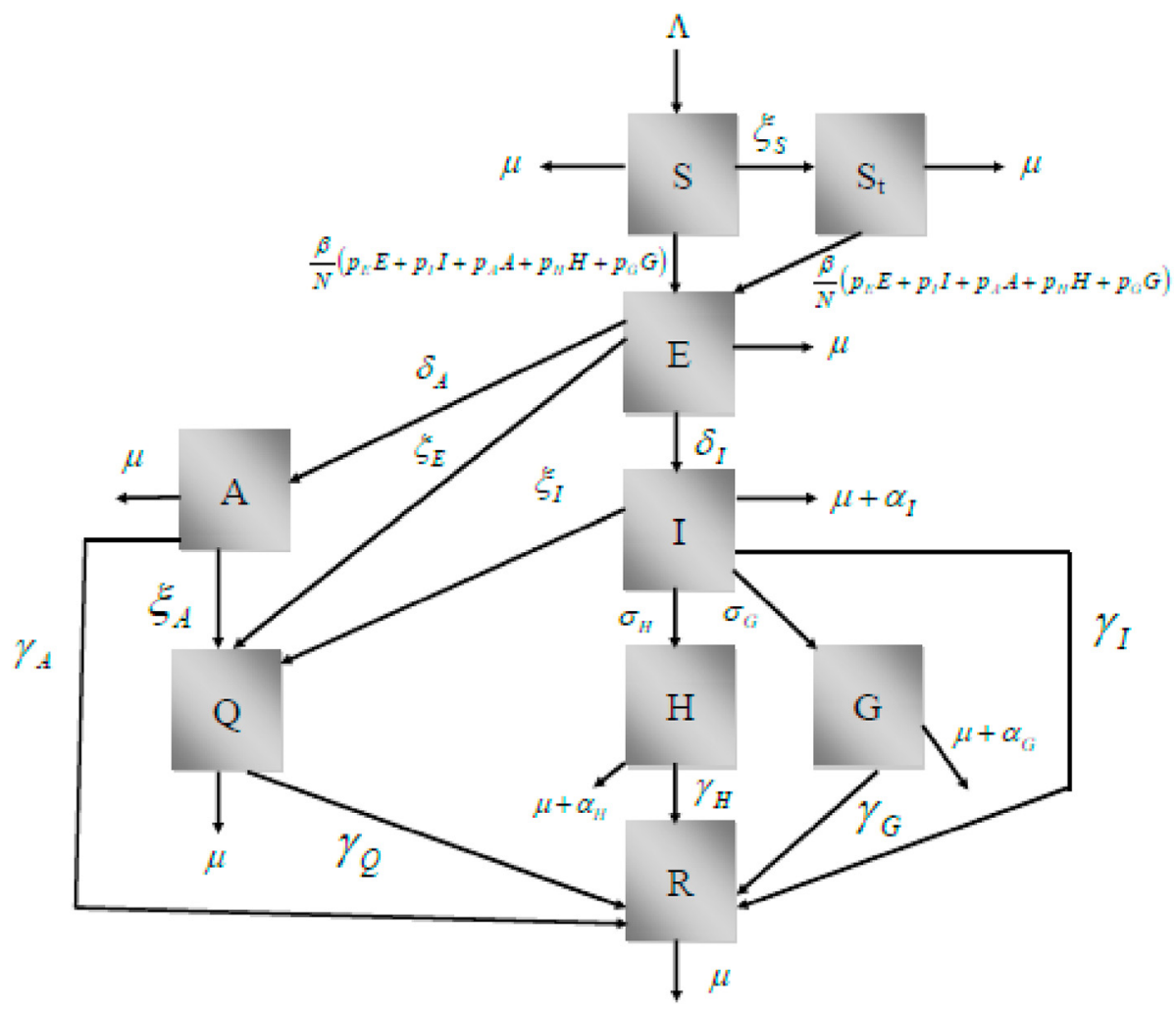

Fig. 1. Schematic representation of the model compartments. 
(i) Susceptible individuals, denoted $S(t)$, grow in number with a birth rate $\Lambda(t)$, and can either die by natural causes with rate $\mu$, or acquire the virus with contact rate $\beta$, or be tested for the SARS-CoV-2, with rate $\xi_{S}$ that begins at a time $t_{i}$ after the beginning of the outbreak.

(ii) Tested susceptible individuals, denoted $S_{T}(t)$, die by natural causes with the same rate $\mu$, or acquire the virus with the same contact rate $\beta$.

(iii) Once infected, the susceptible move to the state of Exposed, denoted $E(t)$. These individuals either die by natural causes with the same rate $\mu$, or evolve to the infectious individuals, denoted $I(t)$, with rate $\delta_{I}$, or beggining at ime $t_{i}$, be tested for the SARS-CoV-2, with rate $\xi_{E}$ or evolve to asymptomatic/oligosymptomatic individuals, denoted $A(t)$, with rate $\delta_{A}$.

(iv) Infectious individuals, $I(t)$, evolve for one of two states: Hospitalized individuals denoted $H(t)$, or to a state in which individuals evolve to and develop severe disease, necessitating respiratory assistance, denoted $G(t)$, with rates $\sigma_{H}$ and $\sigma_{G}$, respectively. Infectious individuals, $I(t)$, also die by natural causes with rate $\mu$, or by the disease, with rate $\alpha_{I}$, and can be tested for the SARS-CoV-2, with rate $\xi_{I}$ that begins at time $\mathrm{t}_{\mathrm{i}}$.

(v) Individuals in the three states, $A(t), H(t)$, and $G(t)$, can die by natural causes with rate $\mu$, or by the disease, with rates $\alpha_{A} \alpha_{H}$, and $\alpha_{G}$, respectively. All indivuduals who acquired the infection and who did not die by the disease recover to a new state, denoted $R(t)$, with rates $\gamma_{I} \gamma_{A}, \gamma_{H}$ and $\gamma_{G}$, respectively.

(vi) Tested individuals who resulted positive for the presence of the virus are quarantined in a stated denoted $Q(t)$. Since these individuals are isolated from the rest of the population, they do not transmit the virus and will eventually recover from the infection,

with rate $\gamma_{Q}$, or will die by natural causes, with rate $\mu$. A small fraction of asymptomatic individuals develop symptoms and are hospitalized with rate $\sigma_{A}$.

(vii) We assumed that the population birth rate $\Lambda(t)$ is equal to the natural mortality of the population, not taking into account the disease-induced mortality.

(viii) The fractions $p_{E}, p_{I}, p_{A}, p_{H}$, and $p_{G}$ of exposed, symptomatic, asymptomatic, hospitalized and severe (ICU patients) individuals can transmit the infection.

The model' states are shown in Fig. 1.

The model's dynamics is described by the following set of differential equations:

$$
\begin{aligned}
& \frac{d S(t)}{d t}=-\beta S \frac{\left(p_{E} E+p_{I} I+p_{A} A+p_{H} H+p_{G} G\right)}{N}-\xi_{S} S \theta\left(t-t_{i}\right)-\mu S+\Lambda \\
& \frac{d S_{T}(t)}{d t}=-\beta S_{T} \frac{\left(p_{E} E+p_{I} I+p_{A} A+p_{H} H+p_{G} G\right)}{N}+\xi_{S} S \theta\left(t-t_{i}\right)-\mu S_{T} \\
& \frac{d E(t)}{d t}=\beta\left(S+S_{T}\right) \frac{\left(p_{E} E+p_{I} I+p_{A} A+p_{H} H+p_{G} G\right)}{N}-\left(\mu+\delta_{A}+\delta_{I}+\xi_{E} \theta\left(t-t_{i}\right)\right) E \\
& \frac{d I(t)}{d t}=\delta_{I} E-\left(\gamma_{I}+\sigma_{H}+\sigma_{G}+\xi_{I} \theta\left(t-t_{i}\right)+\alpha_{I}+\mu\right) I \\
& \frac{d A(t)}{d t}=\delta_{A} E-\left(\gamma_{A}+\xi_{A} \theta\left(t-t_{i}\right)+\mu\right) A \\
& \frac{d H(t)}{d t}=\sigma_{H} I-\left(\gamma_{H}+\alpha_{H}+\mu\right) H \\
& \frac{d G(t)}{d t}=\sigma_{G} I-\left(\gamma_{G}+\alpha_{G}+\mu\right) G \\
& \frac{d Q(t)}{d t}=\left(\xi_{E} E+\xi_{I} I+\xi_{A} A\right) \theta\left(t-t_{i}\right)-\left(\mu+\gamma_{Q}\right) Q \\
& \frac{d R(t)}{d t}=\gamma_{I} I+\gamma_{A} A+\gamma_{H} H+\gamma_{G} G+\gamma_{Q} Q-\mu R \\
& N=S+E+I+R+A+H+G+Q+R+S_{T} \\
& \Lambda=\mu\left(E+I+R+A+H+G+Q+R+S_{T}\right)
\end{aligned}
$$

The Basic Reproduction Number of system (1) is given by:

$$
R_{0}=\frac{\beta \delta_{I}(1+\Omega)}{\left(\mu+\delta_{A}+\delta_{I}\right)\left(\gamma_{I}+\sigma_{H}+\sigma_{G}+\alpha_{I}+\mu\right)}
$$

where: 


$$
\begin{aligned}
\Omega & =p_{E} \frac{\left(\gamma_{I}+\sigma_{H}+\sigma_{G}+\alpha_{I}+\mu\right)}{\delta_{I}}+\frac{p_{A} \delta_{A}}{\delta_{I}\left(\mu+\alpha_{A}+\gamma_{A}\right)}+ \\
& +\frac{p_{H} \sigma_{H}}{\left(\mu+\alpha_{H}+\gamma_{H}\right)}+\frac{p_{G} \sigma_{G}}{\left(\mu+\alpha_{G}+\gamma_{G}\right)}
\end{aligned}
$$

The incidence of infection is given by:

$$
I n c=\beta\left(S(t)+S_{T}(t)\right) \frac{\left(p_{E} E(t)+p_{I} I(t)+p_{A} A(t)+p_{H} H(t)+p_{G} G(t)\right)}{N(t)}
$$

The total number of reported cases is obtained by multiplying the number of infected individuals by a notification ratio $K(t)$ :

$$
\text { Cases }=K(t) \int_{0}^{\infty} \beta\left(S(t)+S_{T}(t)\right) \frac{\left(p_{E} E(t)+p_{I} I(t)+p_{A} A(t)+p_{H} H(t)+p_{G} G(t)\right)}{N(t)} d t
$$

The total number of COVID-19-related deaths is given by:

$$
\text { Deaths }=\int_{0}^{\infty}\left[\alpha_{I} I(t)+\alpha_{H} H(t)+\alpha_{G} G(t)\right] d t
$$

\begin{tabular}{|c|c|c|}
\hline Parameter & Description & Value \\
\hline$\beta(t)$ & Potentially infective contact rate & Fitted (changes over time) \\
\hline$p_{E}$ & Infectivity of exposed individuals & $0.4^{\mathrm{a}}$ \\
\hline$p_{I}$ & Infectivity of symptomatic individuals & $1.0^{\mathrm{a}}$ \\
\hline$p_{A}$ & Infectivity of asymptomatic individuals & $1 / 3^{\mathrm{a}}$ \\
\hline$p_{H}$ & Infectivity of hospitalized individuals & $0.01^{\mathrm{a}}$ \\
\hline$p_{G}$ & Infectivity of ICU patients & $0.01^{\mathrm{a}}$ \\
\hline$\mu$ & Natural mortality rate (life expectancy of 70 years) & $3.91 \times 10^{-5}$ days $^{-1 \text { a }}$ \\
\hline$\delta_{I}$ & Rate of evolution from exposed to infected & $1 / 2$ day $^{-1}$ a \\
\hline$\delta_{A}$ & Rate of evolution from exposed to asymptomatic & $1.45 \mathrm{day}^{-1 \mathrm{~b}}$ \\
\hline$\gamma_{I}$ & Rate of recovery from infected & $1 / 3$ day $^{-1}$ a \\
\hline$\gamma_{A}$ & Rate of recovery from asymptomatic & $1 / 14$ day $^{-1}$ a \\
\hline$\gamma_{H}$ & Rate of recovery from hospitalized & $1 / 10$ day $^{-1}$ a \\
\hline$\gamma_{G}$ & Rate of recovery from ICU & 0.06752 day $^{-1 \mathrm{~b}}$ \\
\hline$\gamma_{Q}$ & Rate of recovery from isolated & $1 / 14$ day $^{-1}$ a \\
\hline$\alpha_{I}$ & Disease-induced mortality rate for infected individuals & $5 \times 10^{-4}$ day $^{-1}$ a \\
\hline$\alpha_{A}$ & Disease-induced mortality rate for asymptomatic individuals & $0^{\mathrm{a}}$ \\
\hline$\alpha_{H}$ & Disease-induced mortality rate for hospitalized individuals & $2.2012 \times 10^{-4} \mathrm{day}^{-1 \mathrm{~b}}$ \\
\hline$\alpha_{G}$ & Disease-induced mortality rate for ICU patients & Fitted (changes over time) \\
\hline$\xi_{S}$ & Testing rate of susceptible individuals & Variable \\
\hline$\xi_{E}$ & Testing rate of exposed individuals & Variable \\
\hline$\xi_{I}$ & Testing rate of symptomatic individuals & Variable \\
\hline$\ddot{\xi}_{A}$ & Testing rate of asymptomatic individuals & Variable \\
\hline$\sigma_{H}$ & Hospitalization rate & $1.973 \times 10^{-2}$ day $^{-1}$ b \\
\hline$\sigma_{G}$ & ICU admission rate & Fitted (changes over time) \\
\hline$K(t)$ & Notification ratio & Fitted (changes over time) \\
\hline$\Lambda(t)$ & Birth rate & Changes over time \\
\hline
\end{tabular}

Finally, the total number of tested individuals is given by:

Table 1

Parameters used in the model.

\footnotetext{
a Assumed

b Fitted.
} 

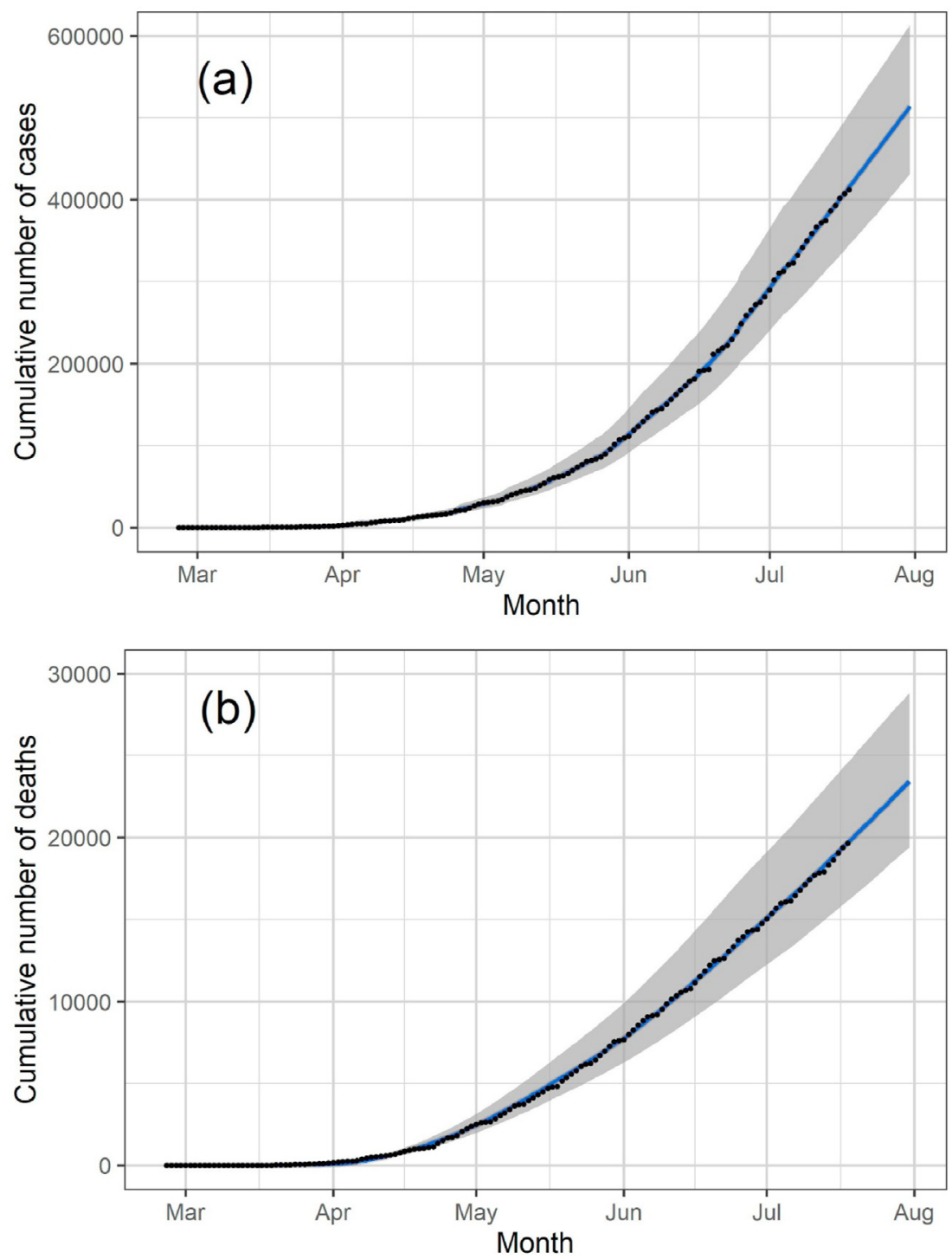

Fig. 2. Cumulative number of reported cases and deaths (black dots in (a) and (b), respectively) and the corresponding fitted model (blue lines). The solid lines and shaded area correspond, respectively, to median values and 95\% probability intervals.

$$
\text { Tested }=\int_{0}^{\infty}\left[\xi_{S} S(t)+\xi_{E} E(t)+\xi_{I} I(t)+\xi_{A} A(t)\right] \theta\left(t-t_{i}\right) d t
$$

\section{Fitting procedure}

Data on the cumulative number of reported cases and deaths were obtained from Seade (Fundação Sistema Estadual de Análise de Dados do Estado de São Paulo). Data on the number of ICU patients were obtained from SIMI (Sistema de Monitoramento Inteligente do Estado de São Paulo). A fitting procedure based on the Levenberg-Marquardt non-linear leastsquares algorithm was used to fit the model's parameters simultaneously to the data on cases, deaths and ICU patients. We used the R package minpack.lm (Elzhov et al., 2016).

We assumed that the potentially infective contact rate, the notification ratio, and the ICU admission rate change every 10 days. (As it will be explained in another paper this simulates the propagation of the disease and compliance with social distancing rules). The parameter values used are shown in Table 1. 


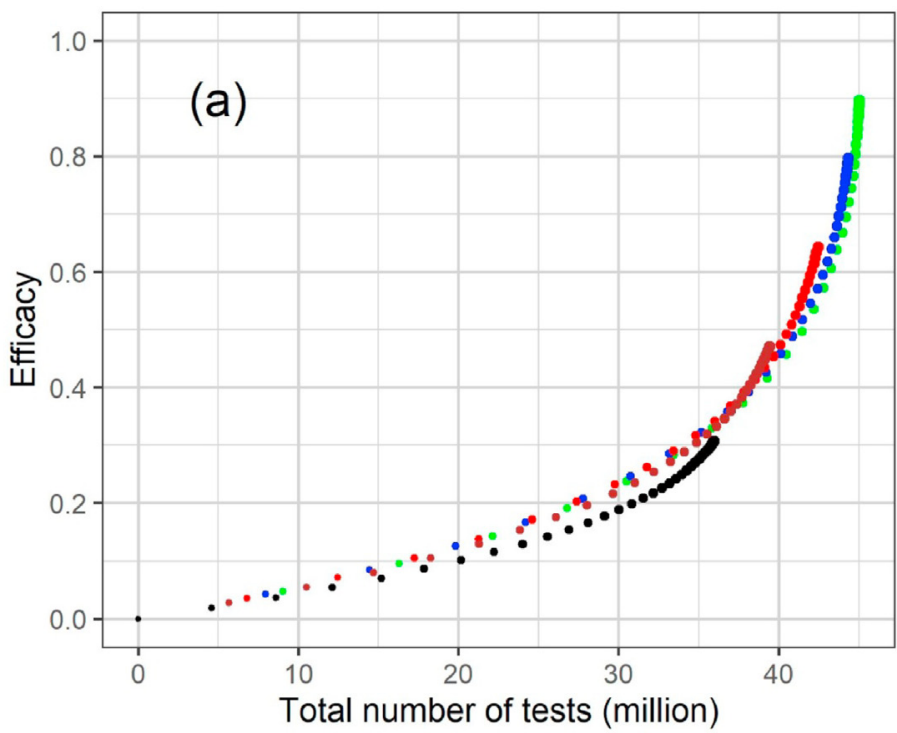

Start

- Apr $1^{\text {st }}$

- May $1^{\text {st }}$

- Jun $1^{\text {st }}$

- Jul $1^{\text {st }}$

- $\operatorname{Aug} 1^{\mathrm{st}}$

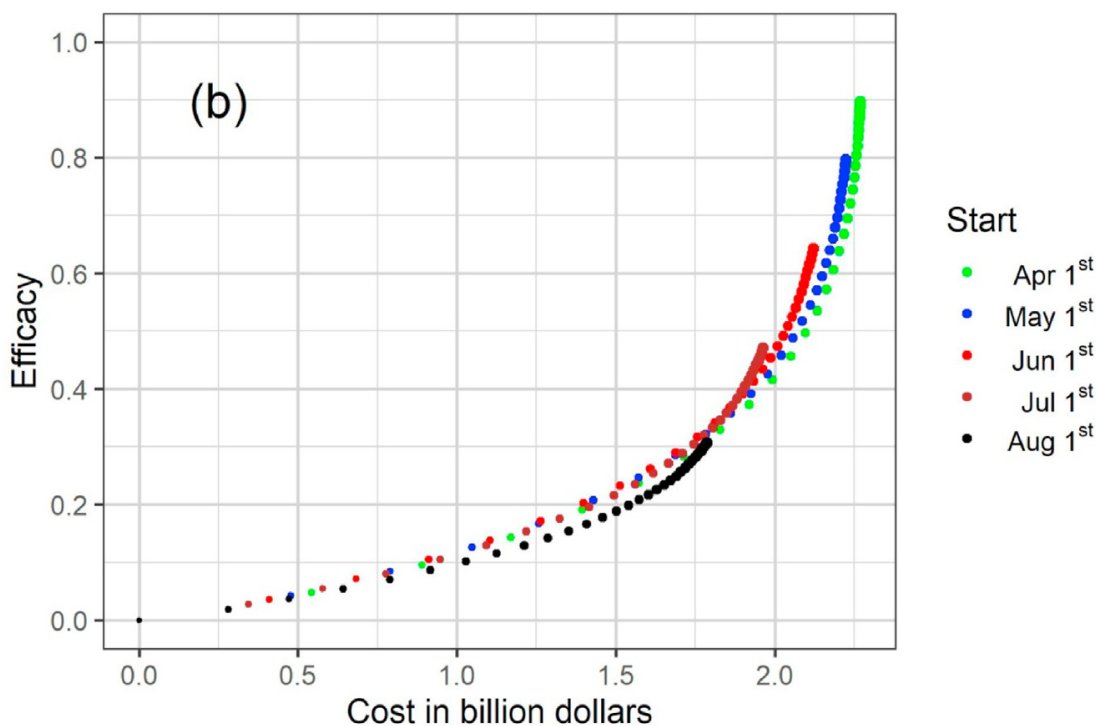

Fig. 3. Results for the strategy that considers testing susceptible and infected (symptomatic and asymptomatic) individuals showing the evolution of the testing strategy efficacy ( 1 minus the number of cumulative cases under a specific testing strategy up to December 31, 2020 divided by the number of cases when no test is used) as a function of the total number of tests (a) and corresponding costs in US dollars (b) for different dates of start. Each dot corresponds to a different daily testing rate and the dot size is proportional to the testing rate.

Model projections for future dates were obtained by keeping fixed the fitted values of the parameters from the last date observed in the data.

\section{The test-trace-quarantine strategy}

Testing, tracing and quarantining positive individuals simulated consists in applying 10 thousand health agents from the programme of primary health of the State Secretary of Health that would visit the 3,933,448 dwellings of the State of São Paulo. In each dwelling, the agent would interview the inhabitants questioning who had had any of the symptoms of COVID19 since March 2020 and about who had had contact to any individual who had the disease in the same period. In case of any positive answer the individuals would be tested for the presence of the virus with the RT-PCR technique. In case of positive result, the individual would be quarantined for 14 days.

The cost of the strategy was calculated assuming the cost of the test, assumed the price tag of US $\$ 48.00$ per sample and the cost of the agenst salary, assumed to be US $\$ 1128.00$ per month. The total cost of each simulated strategy would depend on the rate and of the total number of testing, as well as the duration of each strategy. 


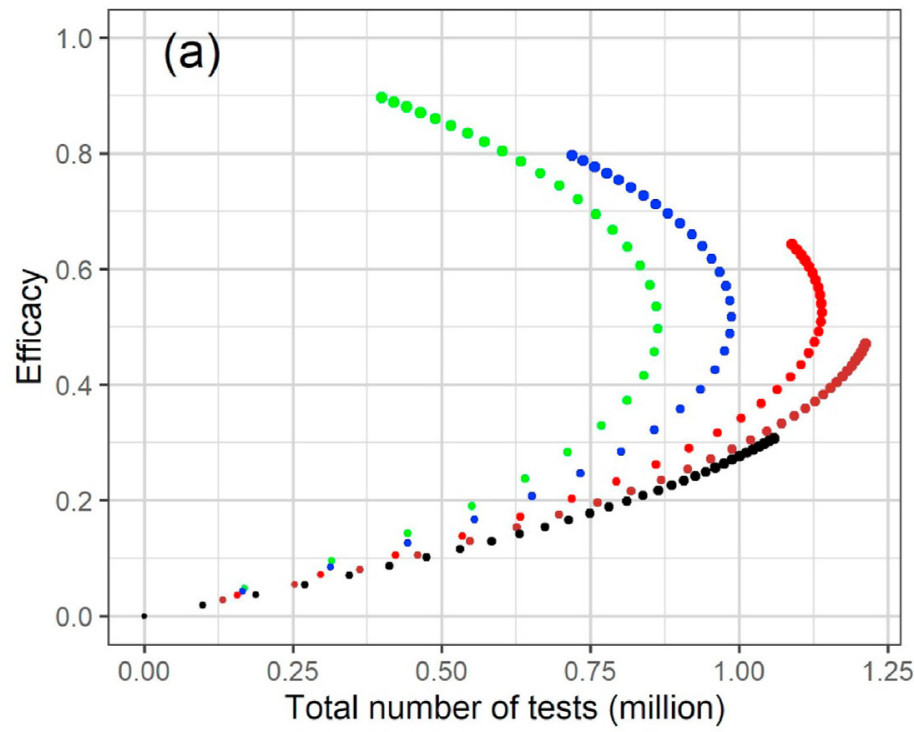

\section{Start}

- Apr $1^{\text {st }}$

- May $1^{\text {st }}$

- Jun $1^{\text {st }}$

- Jul $1^{\text {st }}$

- $\operatorname{Aug} 1^{\text {st }}$

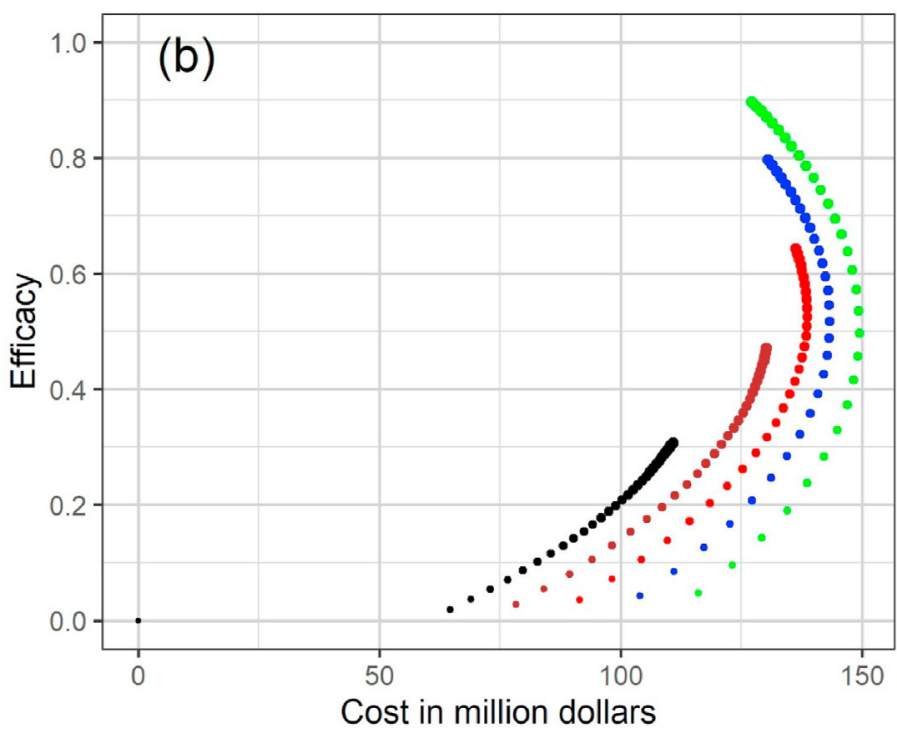

Start

- Apr $1^{\text {st }}$

- May $1^{\text {st }}$

- Jun $1^{\text {st }}$

- Jul $1^{\text {st }}$

- $\quad$ Aug $1^{\text {st }}$

Fig. 4. Results for the strategy that considers testing infected (symptomatic and asymptomatic) individuals showing the evolution of the testing strategy efficacy ( 1 minus the number of cumulative cases under a specific testing strategy up to December 31, 2020 divided by the number of cases when no test is used) as a function of the total number of tests (a) and corresponding costs in US dollars (b) for different dates of start. Each dot corresponds to a different daily testing rate and the dot size is proportional to the testing rate.

\section{Results}

We fitted the model's parameters simultaneously to the data of cumulative number of reported cases and deaths (Fig. 2(a)-(b), respectively), and the number of ICU patients for the state of São Paulo until July 18, 2020. To estimate a 95\% probability interval (shaded area in Fig. 2), we assumed a normal distribution for the contact rate with a standard deviation of 1.5\%. The fitted parameters are shown in Table 1.

The results for the strategy that considers testing susceptible and infected (symptomatic and asymptomatic) individuals are shown in Fig. 3. We calculated a testing strategy efficacy, subtracting from 1 the result of the division of the number of cumulative cases under a specific testing strategy up to December 31, 2020 by the number of reported cases in a scenario in which no test is used. The testing strategy efficacies as a function of the total number of tests and corresponding costs in US dollars are shown in Fig. 3(a)-(b), respectively, for different times $t_{i}$, the strategy started to be applied. We noticed that a 50\% reduction in the number of cases is only achieved when the total number of tests is approximately 40 million (the state population is about 44.6 million people) with an approximate total cost of 2 billion US dollars, except for the start date of August 1st. 


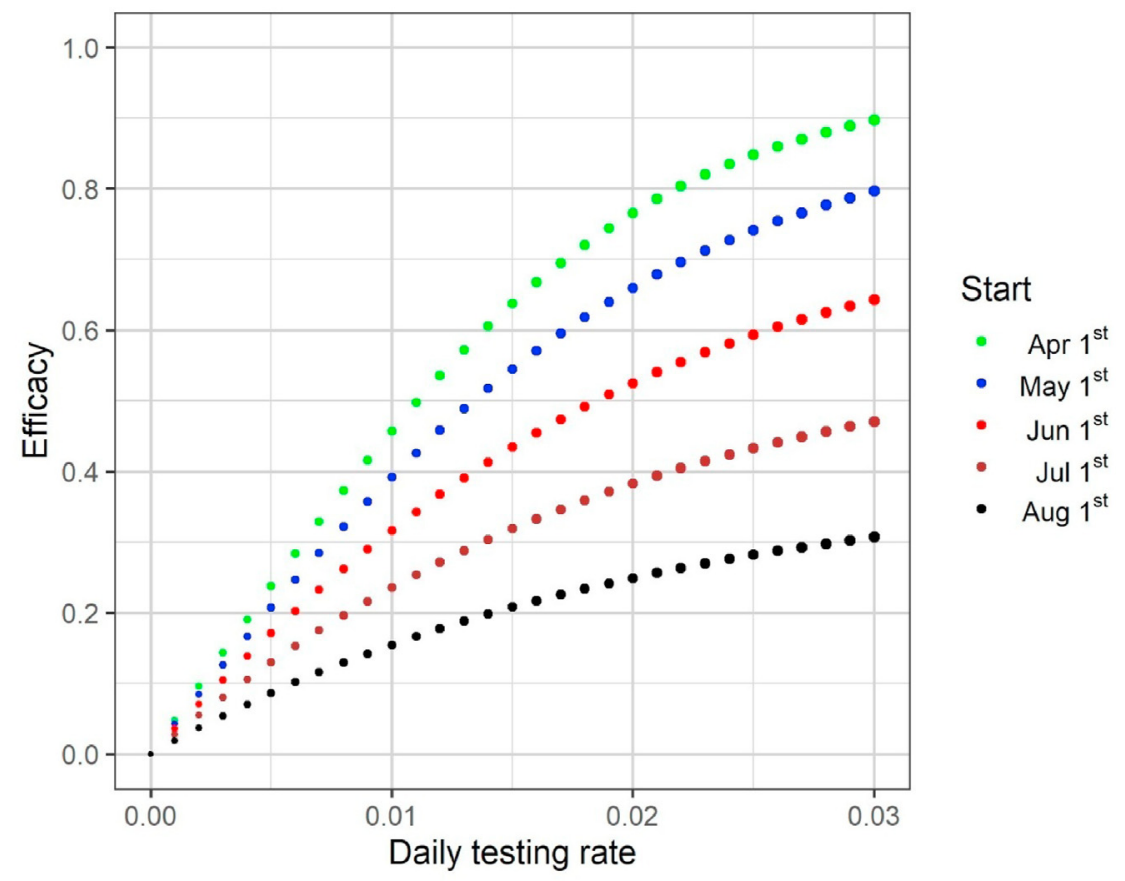

Fig. 5. Results for the strategy that considers testing infected (symptomatic and asymptomatic) individuals showing the evolution of the testing strategy efficacy ( 1 minus the number of cumulative cases under a specific testing strategy up to December 31, 2020 divided by the number of cases when no test is used) as a function of the daily testing rate for different dates of start. The dot size is proportional to the testing rate.

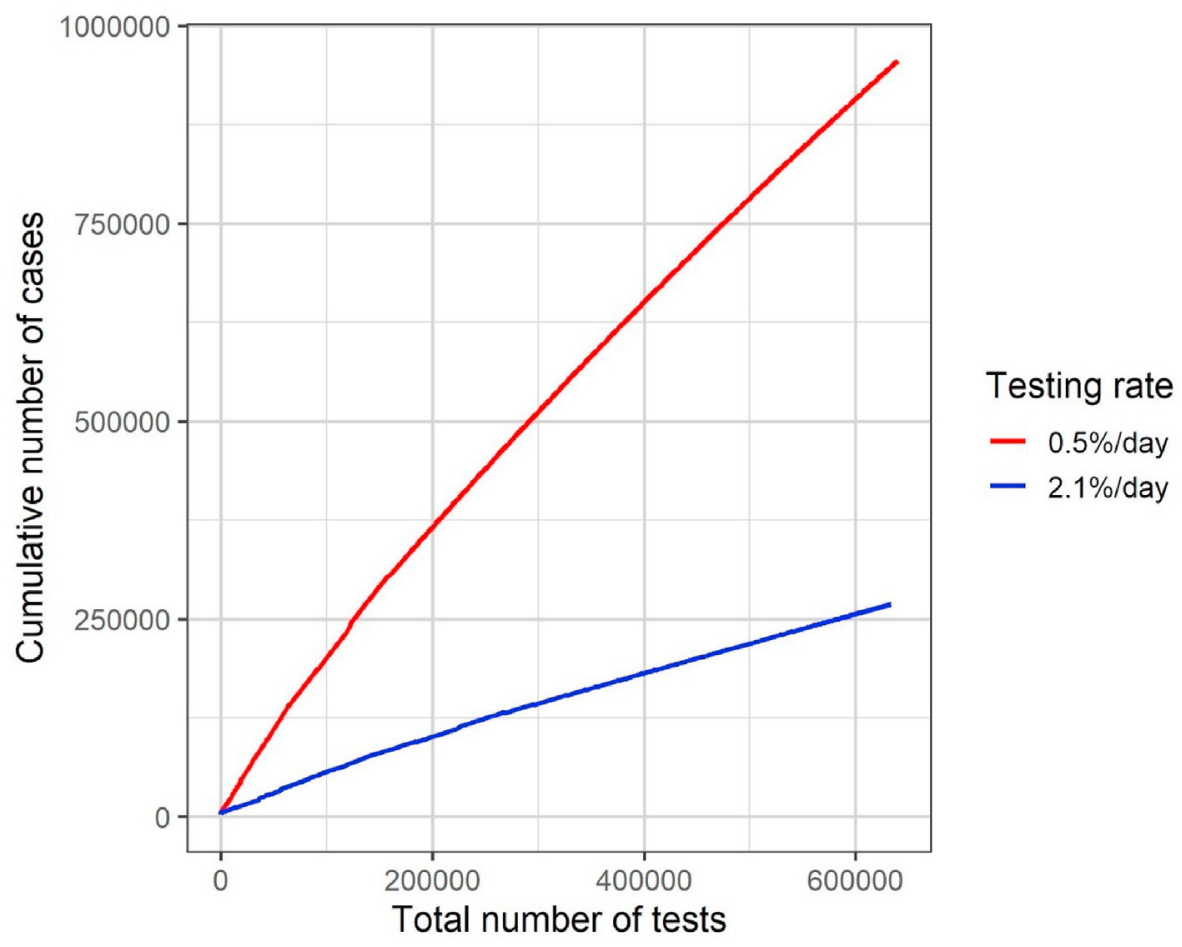

Fig. 6. Total number of tests over time and the corresponding number of cumulative cases for two different daily testing rates $(0.5 \%$ and $2.1 \%$ per day) for mass testing starting on April 1st. 
The results for the strategy that considers testing infected (symptomatic and asymptomatic/oligosymptomatic) individuals are shown in Fig. 4. The testing strategy efficacies as a function of the total number of tests and corresponding costs in US dollars are shown in Fig. 4(a) and (b), respectively, for different dates of start.

In Figs. 3 and 4, each dot corresponds to a different daily testing rate and the dot size is proportional to the testing rate. Dot sizes and corresponding testing rates can be observed in Fig. 5, in which we observe that higher testing rates and earlier start dates are more efficacious.

In Fig. 4(a), dots in the upper part of the graph correspond to higher testing rates. For each date of start, we noticed that dots corresponding to different testing rates are displayed in a concave shape. Thus, depending on the testing rate, similar total number of tests may result in different efficacies. Let us consider the specific case of the testing rates $0.5 \%$ and $2.1 \%$ per day for the start date of April 1st (Fig. 6). We noticed in Fig. 4(a) that the total number of tests used until December 31, 2020, is similar (approximately 0.63 million tests) but the cumulative numbers of cases are about 0.95 and 0.27 million cases for the rates $0.5 \%$ and $2.1 \%$ per day, respectively. In the model, the number of tested individuals is proportional to the number of infected people. For a low testing rate, the initial number of individuals tested and consequently the number of individuals in quarantine are both small, and the force of infection and thus the disease spread are not efficiently reduced. For a high testing rate, on the other hand, the initial number of individuals tested and consequently the number of individuals in quarantine are larger, hence the force of infection and the number of new cases are both reduced more efficiently over time.

When only (suspected) infected individuals are tested, the total cost is higher for earlier start dates (Fig. 4(b)), because operational costs are substantionally higher than the costs related to the diagnostic tests.

\section{Discussion}

We modelled and simulated a test-trace-and-quarantine strategy to control the COVID-19 outbreak in the State of São Paulo, Brasil. The State of São Paulo failed to adopt an effective social distancing strategy, reaching at most 59\% in late March and started to relax the measures in late June, dropping to 42\% in 11 September (Sistema de Monitoramento Inteligente do Estado de São Paulo, 2020). Therefore, São Paulo relies heavily on a massive testing strategy in the attempt to control the epidemic.

We simulated two alternative strategies combined with economic evaluations. One strategy included indiscriminately testing the entire population of the State, reaching more than 40 million people at a maximum cost of 2.25 billion USD, that would reduce the total number of cases by the end of 2020 by $90 \%$. The second strategy investigated testing only symptomatic cases and their immediate contacts - this strategy reached a maximum cost of 150 million USD but also reduced the number of cases by $90 \%$. As we were interested in the simulation of the impact of testing and quarantining in the spread of the infection in the general population, we did not included testing for other clustering. For instance, health workers are tested regularly in Sao Paulo and are isolated when tested positive.

Our model has the following limitations. The model assumes a perfect test with $100 \%$ sensitiviy and specificity; we also assumed that tracing the contacts of the positive cases would be perfect, which is obviously a practical impossibility. Another important limitation of the model is that it does not consider any delay in the results of the test, an important factor that has been limiting this strategy in many places of the world (Kretzschmar et al., 2020). Although the current tests have a delay of many days to present the results, many rapid-molecular point of care tests for detecting positive patients to SARS-CoV-2 are in development (Ahmad et al., 2020), so the delay in test results can be overcome. However, our model does not take into account social inequity in accessing testing (Souza et al., 2020; Martins-Filho et al., 2020). Some strategies could be considered to reduce the testing costs like pooling samples for testing (Abid et al., 2020) or concentrating the tests in neighborhood where sewege systems were tested positive (Foladori et al., 2020).

\section{Conclusion}

Had the State of São Paulo decided to adopt this strategy on April the 1st, it would have been possible to reduce the total number of cases in $90 \%$ at a cost of 2.25 billion US dollars for the indiscriminate strategy but at a much smaller cost of 125 million US dollars for the selective testing of symptomatic cases and their contacts. We conclude that a selective test, trace and quarantine strategy is the most cost effective strategy that could be applied in situations where social distancing is difficult to implement.

\section{Authors contributions}

All authors contributed equally in all phases of this work.

\section{Declaration of competing interest}

The authors declare no conflict of interests. 


\section{Acknowledgements}

This work was partially supported by the project ZikaPLAN, funded by the European Union's Horizon 2020 research and innovation programme under Grant Agreement No. 734584, by LIM01-HFMUSP, CNPq and FAPESP and Fundacao Butantan.

\section{Ethical approval}

Not applicable (this is a purely theoretical work with no human subject involved).

\section{References}

Abid, S., Ferjani, S., El Moussi, A., Ferjani, A., Nasr, M., Landolsi, I., et al. (2020). Assessment of sample pooling for SARS-CoV-2 molecular testing for screening of asymptomatic persons in Tunisia. Diagnostic Microbiology and Infectious Disease, 98, 115125.

Ahmad, S., Ali, N., Kausar, M., Misbah, H., \& Wahid, A. (2020). Road toward rapid-molecular point of care test to detect novel SARS-coronavirus 2019 (COVID19): Review from updated literature. Allergologia et Immunopathologia. https://doi.org/10.1016/j.aller.2020.06.001

Angelo, K. M., Libman, M., Gautret, P., Barnett, E., Grobusch, M. P., Hagmann, S. H. F., et al. (2019). The rise in travel-associated measles infectionsGeoSentinel, 2015-2019. Journal of Travel Medicine, 26. taz046.

Biblioteca Virtual do Estado de São Paulo. (2020). Available from: http://www.bibliotecavirtual.sp.gov.br/temas/sao-paulo/sao-paulo-populacao-do-estado. php. (Accessed 11 September 2020).

Bogoch, I. I., Watts, A., Thomas-Bachli, A., Huber, C., Kraemer, M. U. G., \& Khan, K. (2020). Potential for global spread of a novel coronavirus from China. Journal of Travel Medicine, 27, 1-3. taaa011,.

Candido, D. D. S., Watts, A., Abade, L., Kraemer, M. U. G., Pybus, O. G., Croda, J., et al. (2020). Routes for COVID-19 importation in Brazil. Journal of Travel Medicine, 27. taaa042.

Carmo, R. F., Nunes, B., Machado, M. F., Armstrong, A. C., \& Souza, C. D. F. (2020). Expansion of COVID-19 within Brazil: The importance of highways. Journal of Travel Medicine, 27. https://doi.org/10.1093/jtm/taaa106. taaa106.

Elzhov, T. V., Mullen, K. M., Spiess, A.-N., Bolker, B., \& minpack. (2016). R interface to the levenberg-marquardt nonlinear least-squares algorithm found in MINPACK, plus support for bounds. R package version 1.2-1. https://CRAN.R-project.org/package=minpack.lm.

Foladori, P., Cutrupi, F., Segata, N., Manara, S., Pinto, F., Malpei, F., et al. (2020). SARS-CoV-2 from faeces to wastewater treatment: What do we know? A review. The Science of the Total Environment, 743, 140444.

Halstead, S., \& Wilder-Smith, A. (2019). Severe dengue in travellers: Pathogenesis, risk and clinical management. Journal of Travel Medicine, 26. taz062.

Hellewell, J., Abbott, S., Gimma, A., Bosse, N. I., Jarvis, C. I., Russell, T. W., et al. (2020). Feasibility of controlling COVID-19 outbreaks by isolation of cases and contacts. Lancet Global Health, 8, e488-e496.

Instituto Brasileiro de Geografia e Estatística (IBGE). (2020). Available from: https://www.ibge.gov.br/cidades-e-estados/sp/sao-paulo.html . (Accessed 1 September 2020).

Jesus, J. G., Sacchi, C., Candido, D. D. S., Claro, I. M., Sales, F. C. S., Manuli, E. R., et al. (2020). Importation and early local transmission of COVID-19 in Brazil, 2020 (Vol. 62, p. e30). Revista do Instituto de Medicina Tropical de Sao Paulo.

Kretzschmar, M. E., Rozhnova, G., Bootsma, M. C. J., van Boven, M., van de Wijgert, J., \& Bonten, M. J. M. (2020). Impact of delays on effectiveness of contact tracing strategies for COVID-19: A modelling study. Lancet Public Health, 5, e452-e459.

Martins-Filho, P. R., de Souza Araujo, A. A., Quintans-Junior, L. J., \& Santos, V. S. (2020). COVID-19 fatality rates related to social inequality in northeast Brazil: A neighborhood-level analysis. Journal of Travel Medicine. https://doi.org/10.1093/jtm/taaa128. taaa128.

Massad, E., Amaku, M., Wilder-Smith, A., dos Santos, P. C. C., Struchiner, C. J., \& Coutinho, F. A. B. (2020). Two complementary model-based methods for calculating the risk of international spreading of a novel virus from the outbreak epicentre. The case of COVID-19. Epidemiology And Infection, 148, e109. https://doi.org/10.1017/S0950268820001223

Ministério da Saúde do Brasil. (2020). Painel coronavírus. Avaliable from https://covid.saude.gov.br/. (Accessed 11 September 2020).

Prete, C. A., Buss, L., Dighe, A., Porto, V. B., Candido, D. S., Ghilardi, F., et al. (2020). Serial interval distribution of SARS-CoV-2 infection in Brazil. Journal of Travel Medicine. https://doi.org/10.1093/jtm/taaa115. taaa115.

Salathe, M., Althaus, C. L., Neher, R., Stringhini, S., Hodcroft, E., Fellay, J., et al. (2020). COVID-19 epidemic in Switzerland: On the importance of testing, contact tracing and isolation. Swiss Medical Weekly, 150, w20225.

Sistema de Monitoramento Inteligente do Estado de São Paulo. (2020). Available from: https://www.simi.sp.gov.br/\#/views/PainelCovid-19/ MunicpiosDetalhado?:iid=1. (Accessed 11 September 2020).

Souza, C. D. F., Carmo, R. F., \& Machado, M. F. (2020). The burden of COVID-19 in Brazil is greater in areas with high social deprivation. Journal of Travel Medicine. https://doi.org/10.1093/jtm/taaa145. taaa145.

Tuite, A. R., Watts, A. G., Khan, K., \& Bogoch, I. I. (2019). Ebola virus outbreak in North Kivu and Ituri provinces, Democratic Republic of Congo, and the potential for further transmission through commercial air travel. Journal of Travel Medicine, 26. taz063.

Wilder-Smith, A., Chang, C. R., \& Leong, W. Y. (2018). Zika in travellers 1947-2017: A systematic review. Journal of Travel Medicine, 25 , tay044.

Wilder-Smith, A., \& Freedman, D. O. (2020). Isolation, quarantine, social distancing and community containment: Pivotal role for old-style public health measures in the novel coronavirus (2019-nCoV) outbreak. Journal of Travel Medicine, 27. https://doi.org/10.1093/jtm/taaa020. taaa020. 